\title{
Temperature Control on FPGA using SPI Protocol with Thermopar Max6675k
}

\author{
Edwar Jacinto G. ${ }^{1}$, Fredy H. Martínez S. ${ }^{2}$, Holman Montiel A. ${ }^{3}$ \\ Universidad Distrital Francisco José de Caldas, Facultad Tecnológica \\ Cll 68 D Bis A Sur No. 49F - 70, Bogotá D.C., Colombia \\ 1ejacintog@udistrital.edu.co, ${ }^{2}$ fhmartinezs@udistrital.edu.co \\ ${ }^{3}$ hmontiela@udistrital.edu.co
}

\begin{abstract}
This paper shows the design of a temperature control for a water heater using a digital controller on an FPGA with SPI communications protocol, which makes use of a thermo-resistance with alternating current $(110 \mathrm{v}-60 \mathrm{~Hz})$ to heat water from the room temperature to its boiling point. The Dimmer that has a Triac BTA16-600B works with control values between 3.3-5 Volts with a charge value for up to 220 Volts AC and peak currents of 5 Amps, the temperature sensor is a K-type thermocouple coupled to a Cold junction Max 6675K compensator.
\end{abstract}

Keyword-FPGA, Digital Control, Temperature control, Measuring and instrumentation.

\section{INTRODUCTION}

Currently the work of electronic instrumentation has changed radically, since the integration of signal conditioning circuits [1], make it not only required to perform the digital readings by a communication bus [2], but also to design robust systems that allow centralizing information through a field bus between different programmable digital devices and thus have control of all system variables. [3-4]

At present, there is a strong tendency to use programmable logic devices type FPGA to perform the calculations of various digital controllers [5], since they can perform the necessary calculations concurrently; what makes them ideal in applications that require a high response speed [6], dynamic shift of the sampling period [7], in addition to performing tasks of communication and visualization of the system variables in a parallel way in a graphical interface [8].

The systems of data acquisition and temperature variable control are widely used in various industrial and productive applications, ranging from a simple temperature control of a tank, machines for precision metal welding [9], chemical plants [10], until the temperature control of one of the modules of a spaceship [3]. In general terms, you can find a large number of applications that carry out processing tasks with fairly short sampling and capture times [11], making corrections to drift errors, errors due to Gaussian noise, delays due to acquisition time, correction by cold juncture, etcetera. [12]; procedures that were performed with analog circuits, but nowadays are performed with sensors packed in the same silicon module that performs the work of conditioning and transmission of information.

In addition to the process of signal acquisition and controller calculation, linearization and signal filtering tasks are required [13-14], along with the possibility of performing control systems such as PID, Fuzzy Logic [15] or another digital technique that fits the needs of the system, even could be used auto-tuning techniques applicable in an FPGA-type device to some of the aforementioned control techniques.

\section{METHODOLOGY}

\section{A. Cold junction compensator Max $6675 \mathrm{~K}$}

The operations of a thermocouple are based on the energy change in which there is a flow of energy from the area of higher temperature to lower temperature, in the union of the two metals that this thermocouple is formed a potential difference between the ends which make up the metals and varies depending on the difference in temperatures. The responses of these measurements depends on the nature of these metals and their manufacture, the part that comes into contact with the element to be measured is called hot junction which is directly the measurement that is of interest for the operation of the system. The tips of the elements where the measurement is taken electrically are called cold junction. The equation that describes this electrical behavior is given by:

$$
\text { Vtermopar }=\alpha *\left(T^{\alpha}-T o\right)=\alpha * T^{\alpha}-\alpha * \text { To }
$$

Where $\alpha$ is a parameter dependent on the types of materials used for its manufacture and gives the sensitivity of the tension at the exit of the cold junction, To is the ambient temperature where the measurement will be made and $T^{\alpha}$ the temperature of interest. [16] 
When the measured values are acquired, the values of the thermocouple (hot junction) and the ambient value (cold junction) must be taken into account, however with the variation of the ambient temperature it is logical to think that the temperature value or the voltage output change. With the cold junction compensator, in this case, the use of the Max $6675 \mathrm{~K}$ module, its main function is the elimination of the ambient temperature, this is done by adding a cancellation variable to the equation that describes the measurement behavior, this term is, $\alpha^{*}$ To physically create an auxiliary circuit that depends on the same ambient temperature to operate and delivers a cancellation voltage to that created by the cold junction, see Fig. 1.

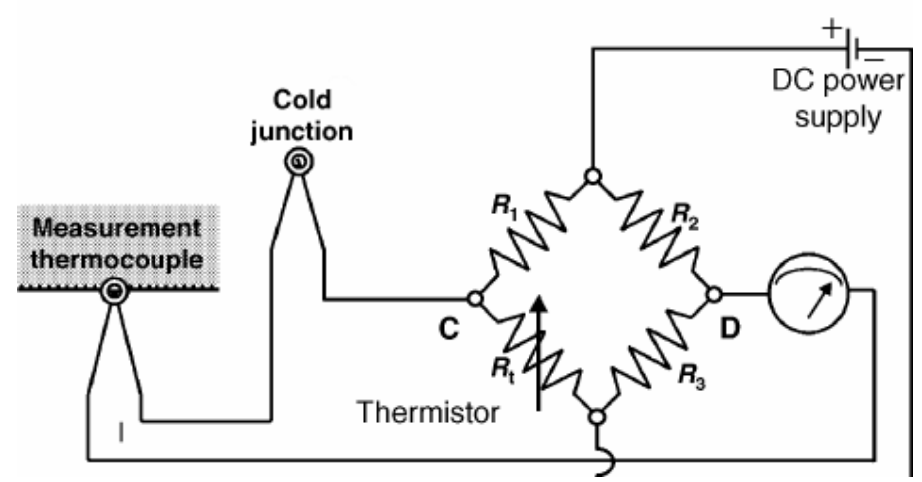

Fig1. Basic cold junction compensation circuit [17]

The main element of the compensating circuit is a thermistor dependent on the ambient temperature, based on a semiconductor that varies its response depending on the temperature, the output voltage after the rectification is expressed as:

$$
\text { Vtermistor }=\beta * \text { To }
$$

Where $\beta$ is precisely the sensitivity of the circuit and the equation of the voltage divider is given by:

$$
\text { Vcompensador }=\text { VR } 1=\text { Vtermistor } * \frac{R 1}{R 1+R 2}=\beta * \text { To } * \frac{R 1}{R 1+R 2}
$$

The compensation is as simple as getting a voltage value with the Vtermopar quantity to cancel the effect of the cold junction, in the meanwhile:

$$
\text { Vtermopar }=\alpha * T^{\alpha}-\alpha * T o * \frac{R 1}{R 1+R 2}
$$

The condition to fulfill is:

$$
\beta * T o * \frac{R 1}{R 1+R 2}=\alpha T o
$$

The cold junction compensator Max $6675 \mathrm{k}$ is in its module a digital analog converter specialized to work with type $\mathrm{k}$ thermocouples, which consists of the $\mathrm{NiCr}-\mathrm{Ni}$ or $\mathrm{NiCr}-\mathrm{NiAl}$ material combinations; this thermocouple in working with the cold junction compensator Max $6675 \mathrm{~K}$ has a measurement range from $0^{\circ}$ to $1024^{\circ}$ Celsius with a resolution of 12 bits, $0.25^{\circ}$ Celsius. The SPI communication protocol uses the 12 bits for unidirectional data communication and discards mainly the most significant bit, since it only works in the range of positive values. The temperature conversion times range between $170 \mathrm{~ms}$ and $220 \mathrm{~ms}$, in order to have a clear and concise measurement with its measurement time value it is recommended to use a longer time than the upper one, in this case it is recommended to request the sensor data $250 \mathrm{~ms}$ between measurement and measurement.

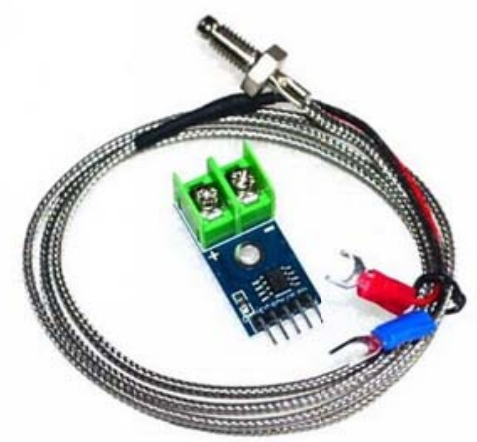

Fig 2. Max6675K with thermo pair type K 


\section{B. SPI Protocol (Serial Peripheral Interface)}

The SPI serial communications protocol was born in the early 1980s with the effort of Motorola as one of the most popular work protocols for its speed of transmission, simplicity and easy operation with many devices, its application can range from screens to microcontrollers.

The synchronous protocol works in full duplex mode to transmit and receive information, being able to communicate bi-directionally and unidirectionally with the devices that support this type of communication through a direct line so that the entire process is synchronous.

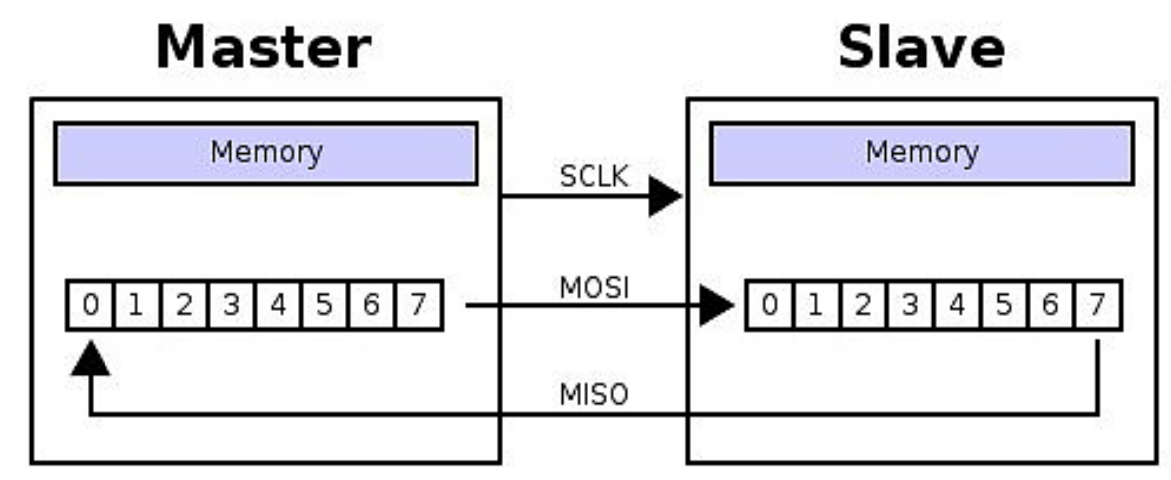

Figure 3. Basic Master-Slave structure

The protocol always defines a master (Master) which is responsible for transmitting and receiving information to its connected elements (slaves), it should be noted that for the Master to receive information a second shift register is necessary, one assigned to the Master and one for the Slave, this is because the storage of bits is clearly done in parallel to then a serial conversion and thus the arrival of information.

The 4 types of logical lines are, MOSI (Master out salve in), MISO (Master in slave out), CLK and SS. The first two are used for master-slave and slave-master communication. CLK is the synchronous line that comes from the master and is used to precisely synchronize the sending and receiving of data between the elements of the system. And finally the SS line when working with more than one slave is responsible for the selection of the slave in the system.

\section{IMPLEMENTATION AND RESULTS}

\section{Reading the MAX6675 Sensor using the FPGA}

Fig. 4 shows some sections of the Max6675 sensor reading using the SPI protocol through the FPGA, in the first instance a driver was made to read the data and display them in a seven segment display, using a Binary to BCD converter generalized for N-bits.

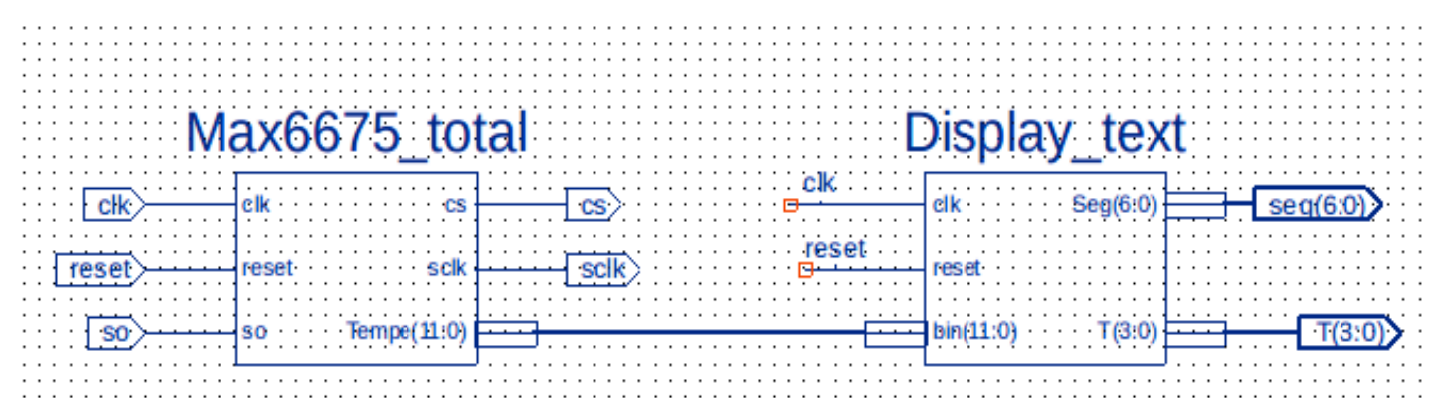

Figure 4. Basic block diagram of Max6675 sensor reading by SPI

To perform the reading of the sensor, a finite state machine FSM was designed and implemented, see Fig. 5 (a), which performs the management of the SPI protocol together with the control of the sampling frequency of the signal acquisition system of temperature, see Fig. 5(b). 


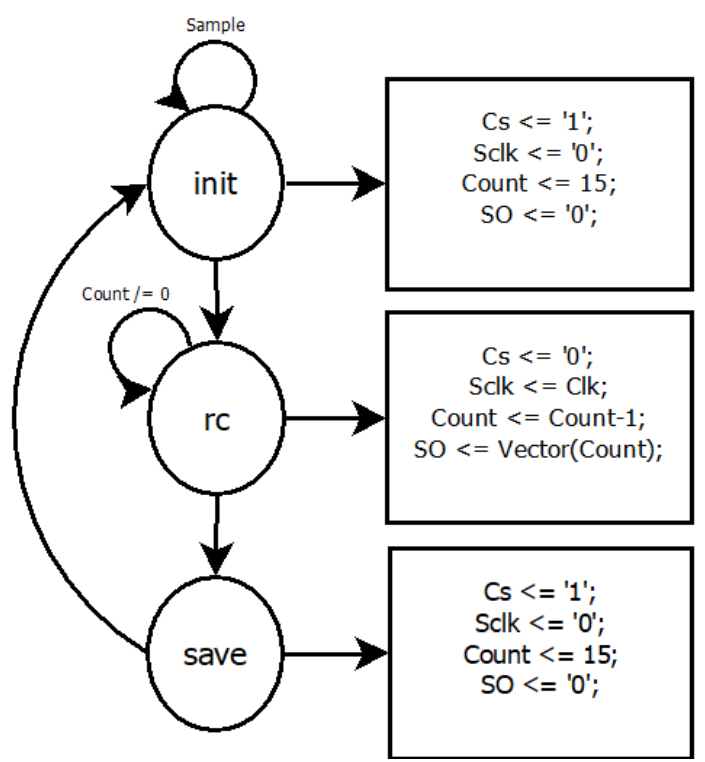

a)

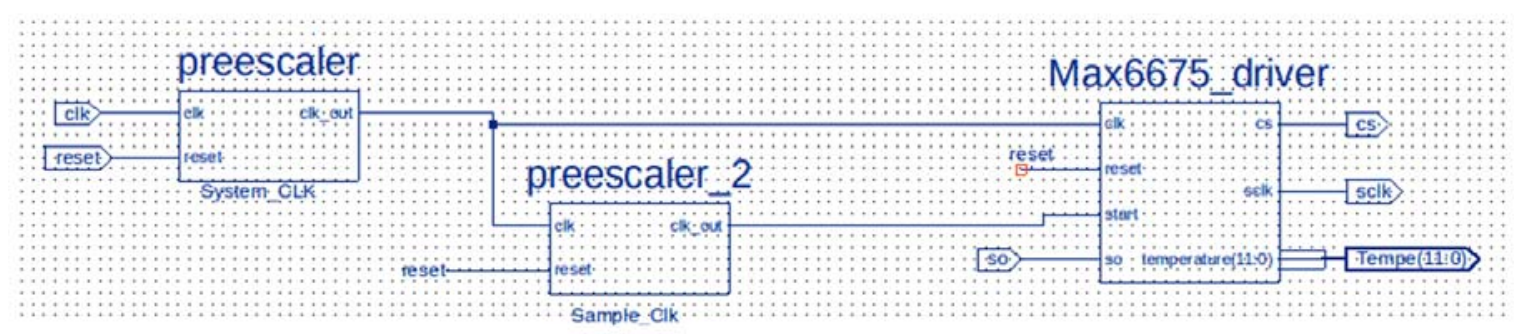

b)

Figure 5. (a) State diagram (b) Diagram of blocks

Modeling, design and implementation of digital control over the FPGA

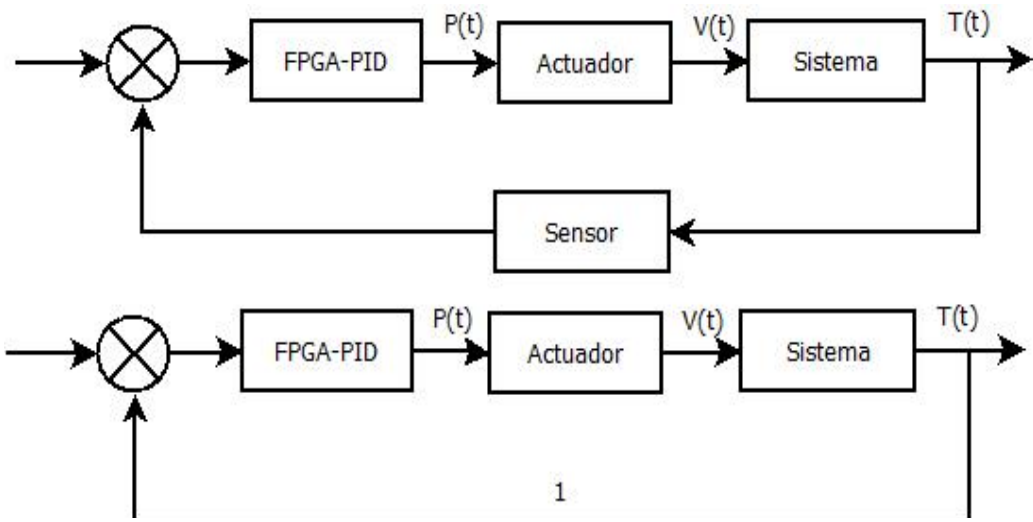

Figure 6. Loop Diagram closed 1 and 2

The closed loop system in this case can be described as a feedback system with gain 1, since the sensor directly delivers the measured values and it is not necessary to convert and manipulate the data delivered by it.

The data delivered by the FPGA, which were subsequently transmitted under the SPI communication, are analyzed and validated by the system under a PID controller, are delivered to the actuator under a temperature value set by the user. The PID controller under a compensation coefficient and with a peak over no more than $5 \%$ guarantees a heating speed in the shortest possible time; it is a system that guarantees a non-saturation of the Dimmer.

The response in open loop was found with the measurement of heating times until these measurements reached desired constant values. The Matlab identifier gives us a graph of temperature vs. time, see Fig. 7, in which it can be seen that the maximum stable value is around 92 degrees, which is the boiling value of water at the height of the city in the that the tests were done. 


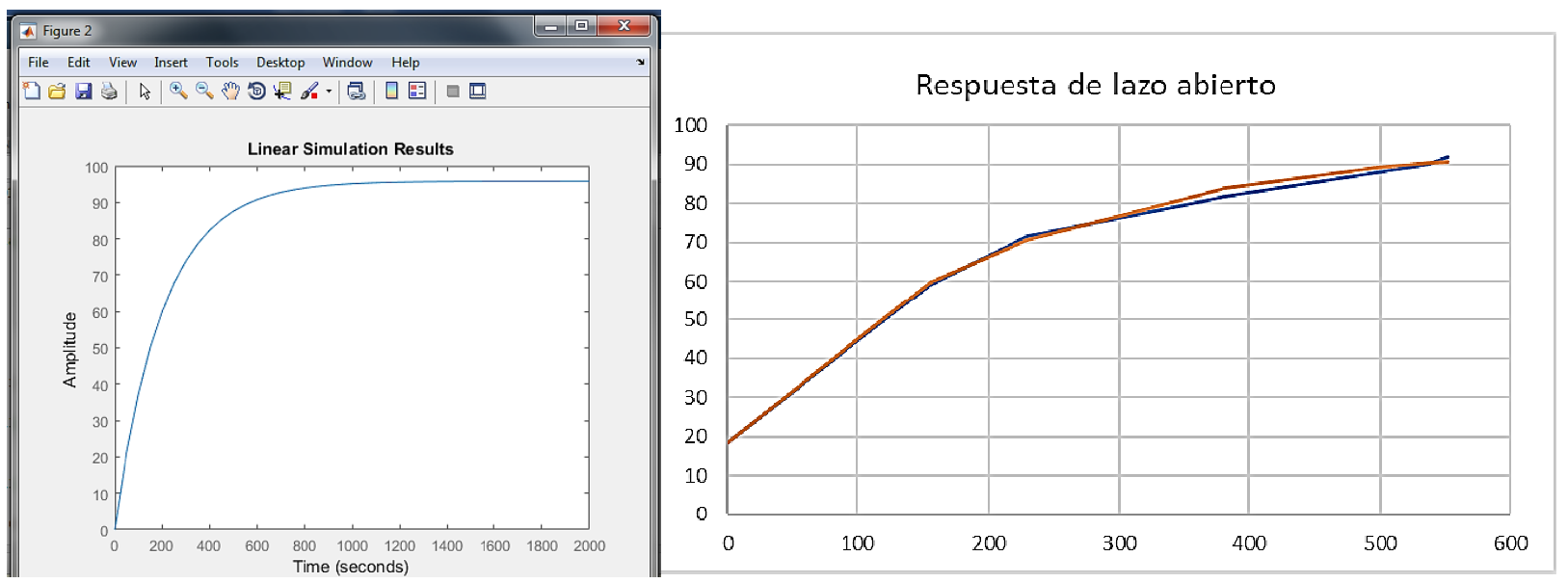

Figure 7. Matlab and Excel open-loop response

Obtaining the data gives us a growth value of 240 seconds before reaching a stable value; the blue line represents the response obtained with real values measured with a thermocouple and a computational timer; under it, the response obtained with coefficients that represent this behavior mathematically is found in orange. The tool "rltool" gives us a mathematical representation of poles, zeros and in this case the limit of the compensator value for a peak envelope of no more than $5 \%$, in addition to a view of the compensation values and the attempt of an equation of transfer function between the input in $\mathrm{AC}$ voltage values and the output in terms of temperature. The poles and zeros of the PID controller are shown below.

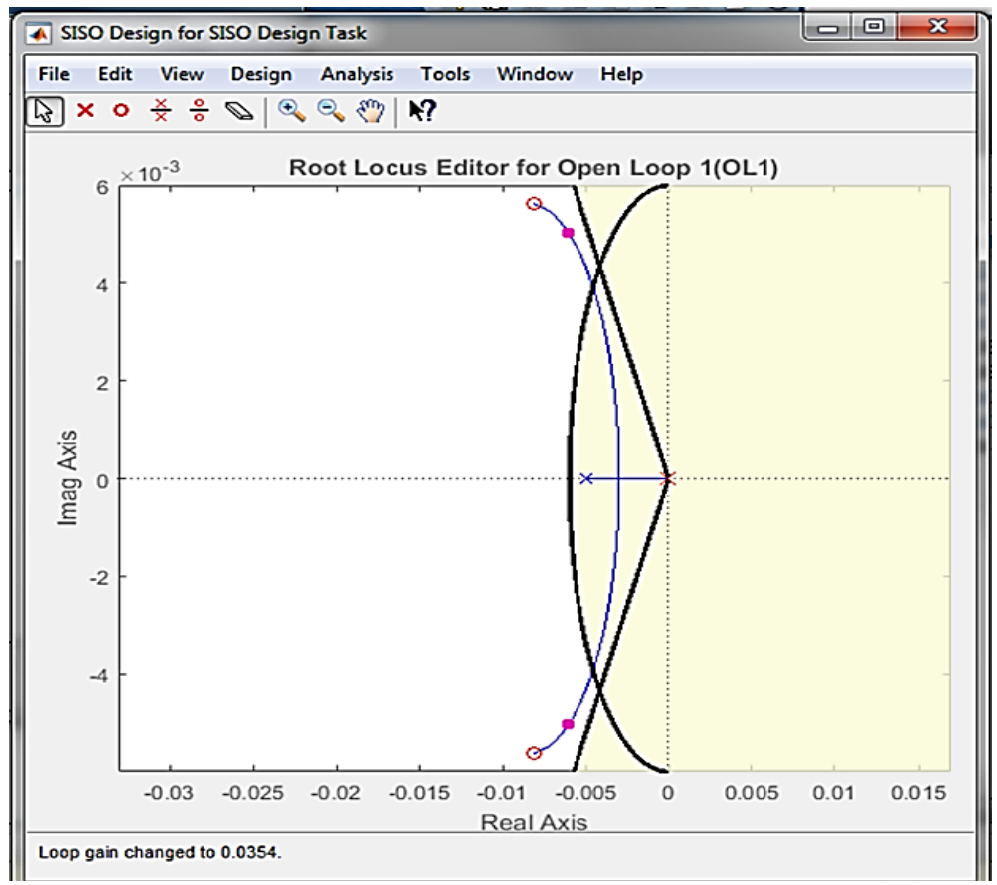

Figure 8. Root Locus, Poles, Zeros in PID

In Fig. 8 we can see the $\mathrm{V}$ shape where the maximum no-peak envelope of $5 \%$ is represented, limiting the value of the compensator and the regions where we can define our zeros and therefore the region for the aforementioned compensator coefficient. The type of control used was a PID controller. During his choice, this type of control was defined due to its low saturation in terms of compensation value at the Dimmer output. 


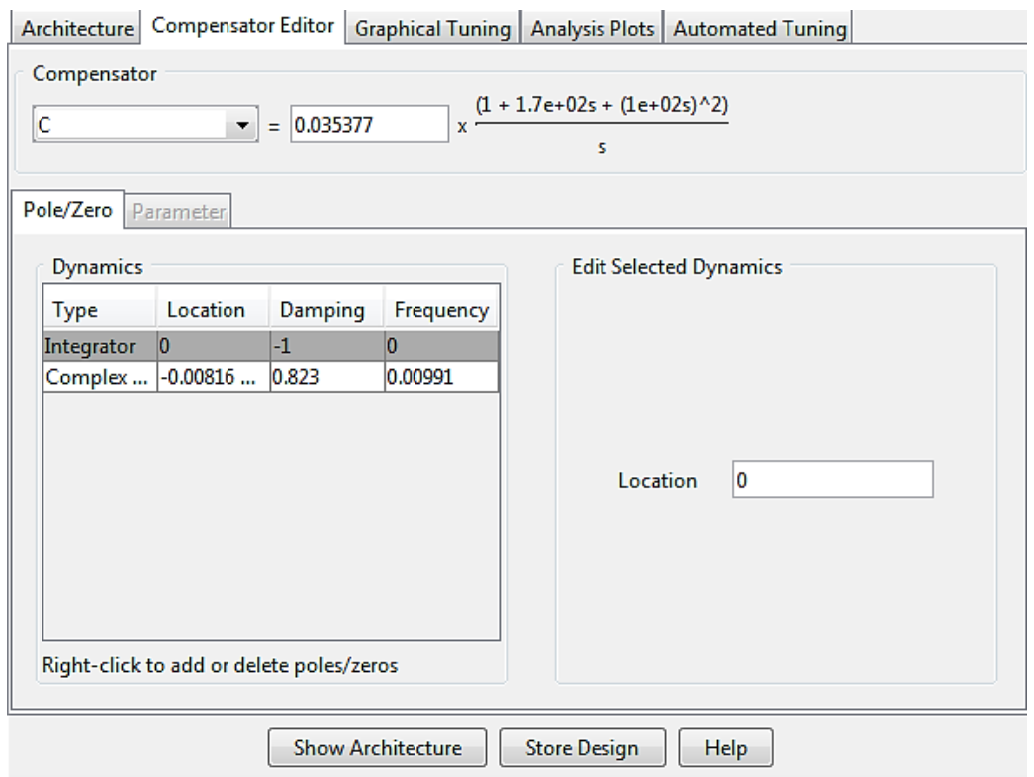

(a)

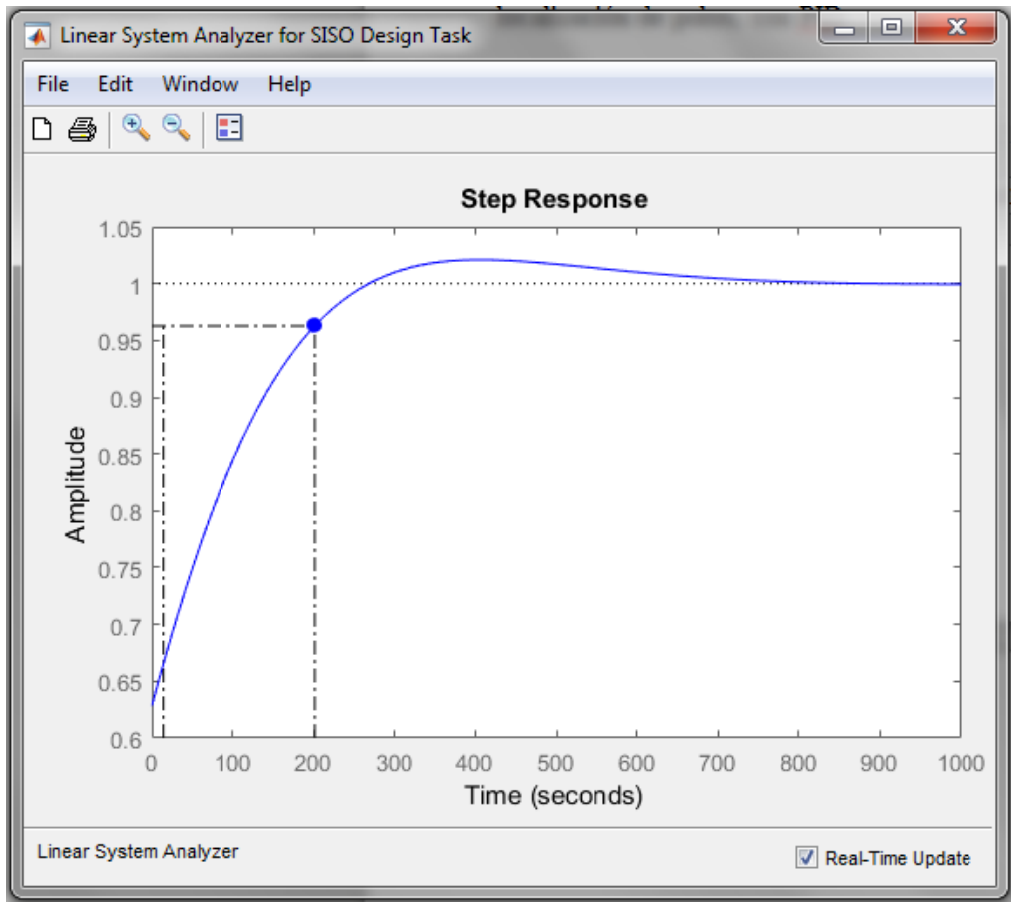

(b)

Figure 9. (a) Controller coefficients (b) control response

The compensation coefficient is observed along with its characteristic equation and below the coefficients that describe the behavior. In Fig. 9 it can be seen a stabilization time of 700 seconds and a maximum rise point of 200 seconds, with a rise peak of no more than $5 \%$. In the export of the controller and then with the conversion to a discrete equation it is obtained the following equation. 


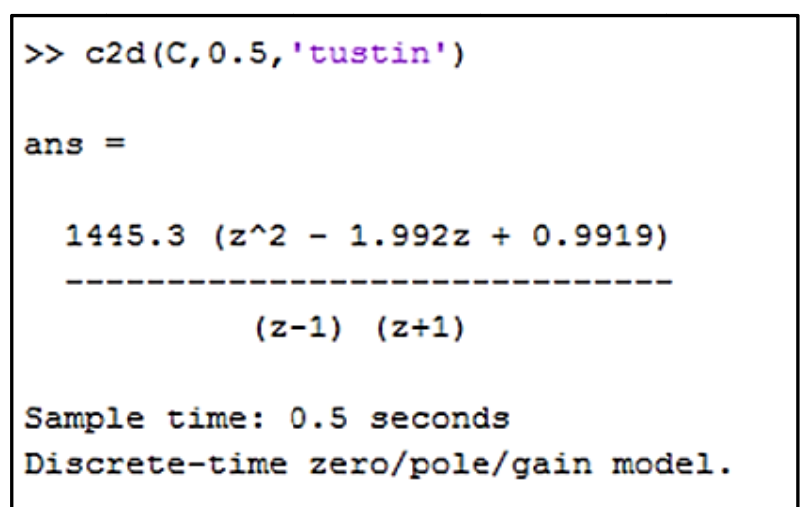

Figure 10. Controller discretized equation

For the conversion to a discrete equation, the frequency was declared at $2 \mathrm{~Hz}$ since the refresh value at the output of the cold junction compensator is $4 \mathrm{~Hz}$, thus guaranteeing a new value whenever a controller data is obtained. The equation in terms of $\mathrm{P}(\mathrm{n})$ is:

$$
\begin{gathered}
C(z)=\frac{1445.3\left(z^{2}-1.992 z+0.9919\right)}{(z-1)(z+1)}=\frac{P(z)}{T(z)} \\
\frac{z^{-2}}{z^{-2}} * \frac{1445.3\left(z^{2}-1.992 z+0.9919\right)}{(z-1)(z+1)}=\frac{P(z)}{T(z)} \\
\frac{1445.3\left(1-1.992 z^{-1}+0.9919 z^{-2}\right)}{1-z^{-2}}=\frac{P(z)}{T(z)} \\
1445.3 T(z)-2879.0376 T(z) z^{-1}+1433.59307 T(z) z^{-2}=P(z)-P(z) z^{-2} \\
1445.3 T(n)-2879.0376 T(n-1)+1433.59307 T(n-2)=P(n)-P(n-2) \\
P(n-2)+1445.3 T(n)-2879.0376 T(n-1)+1433.59307 T(n-2)=P(n)
\end{gathered}
$$

The discrete operation and the behavior of the measurement system together provide a simple measurement when performing work in environments with little time to work. The variable configuration of the desired temperature and the maximum peak envelope increase its versatility to work in ranges from $0^{\circ}$ to $1024^{\circ}$ Celsius, which are allowed by the cold junction compensator and type $\mathrm{K}$ thermocouple.

\section{CONCLUSIONS}

The implementation of a PID controller was made, seeing the behavior of the different types of P, PD and PID controllers, a controller with a low compensation value is obtained that does not saturate the output of the PWM to control the Dimmer of the system. This control allows defining the peak overheating, which makes it one of the most dynamic controls to work in this type of systems with slow response.

For the implementation of the reading of the sensor and the digital controller, less than $4 \%$ of the total resource of a Xilinx Spartan 3E FPGA of one hundred thousand gates was used, a state machine was made to perform the digital readings, using only $2 \%$ of the sequential and combinatorial digital resources available for this low cost FPGA; In addition to this, the maximum delay time of both the controller and the driver that reads the sensor data is only 2.45 nanoseconds, which means that the sampling time of the controller could be up to $408 \mathrm{Mhz}$, result that would allow drivers with higher sampling times or multi-frequency techniques or simply to make a single controller with so little resource, that could perform up to 20 controls in parallel of different processes that require temperature measurement without any inconvenience.

\section{ACKNOWLEDGMENT}

This work was supported by the Universidad DistritalFrancisco José de Caldas FacultadTecnológica. The views expressed in this paper are not necessarily endorsed by District University. The authors thank the research group ARMOS for the evaluation carried out on prototypes of ideas and strategies 


\section{REFERENCES}

[1] D. Y. Scientist y A. T. Conversion, "Three Channel Temperature Sensing Node for Sensor on Bus Approach".

[2] D. Yadav y S. Mani, "Three Channel Temperature Sensing Node for Sensor on Bus Approach", CARE, 2013.

[3] H. K. Singh, A. Chauhan, S. Sreelal, S. Sreeekumar, y A. B. Diagram, "High-capacity Data Acquisition System based on Cortex-M3 for Aerospace Applications", núm. 978, 2017.

[4] T. Pozna, "Raspberry Pi as a Measurement System Control Unit", pp. 2-5, 2014.

[5] K. Lin et al., "A Design of Constant Temperature Control System in 3D Printer", pp. 30-31, 2016.

[6] J. Wu, L. Zhou, P. Sun, y X. Du, "Control of IGBT Junction Temperature in Small- Scale Wind Power Converter", núm. 51137006, 2014.

[7] K. K. Mahant, A. V Patel, A. Vala, y R. Goswami, "FPGA based Temperature Control and Monitoring System for X-ray Measurement Instrument", pp. 3249-3252, 2016.

[8] A. Beerelli, P. Chandra, S. Arunkumar, y M. Venkatesan, "Automated Temperature Control in Batch Reactor using LabVIEW", 2014.

[9] A. M. Abdul-wahed, A. L. Roy, y K. Takahata, "Microplasma Drawing of Thermocouple Sensors", pp. 1-3, 2016.

[10] A. Uart, "Real Time Controlling Of the Critical Parameters with Time and Power Efficient Model in the Refinery", pp. 1-5.

[11] L. Wang, G. Liu, y J. Wang, "A Method of Temperature Drift Compensation for Pulse Synchronization in High- Speed Signal Acquisition", 2017.

[12] A. Mukherjee, D. Sarkar, A. Sen, D. Dey, y S. Munshi, “An Analog Signal Conditioning Circuit for”, CARE, 2013.

[13] K. Pal, S. Rana, V. Kumar, A. K. Dagar, A. Chandel, y A. Kataria, "FPGA Implementation of Steinhart - Hart Equation for Accurate Thermistor Linearization", vol. 18, núm. 6, pp. 2260-2267, 2018.

[14] M. Engin, "Compensation of Thermocouple Nonlinearities with", pp. 234-237, 2016.

[15] O. Grairi, "Design and Implementation of a Versatile Display System based on FPGA for Embedded Systems", pp. 2-6, 2015

[16] J. L. Marcano, "Medidor de temperatura con Termopar", 2008. [En línea]. Disponible en: http://iindustrial.obolog.es/medidortemperatura-termopar-86703. [Consultado: 05-oct-2018].

[17] C. Camci, "Cold junction compensation", 2010. [En línea]. Disponible en: https://www.researchgate.net/figure/Cold-junctioncompensation-of-a-thermocouple_fig4_228017847. 